\title{
Perfil clínico, prevalência e mortalidade de pacientes hospitalizados com doenças hepáticas crônicas infectados com SARS-COV-2: Uma revisão integrativa
}

\author{
Clinical profile, prevalence and mortality of hospitalized patients with chronic liver diseases \\ infected with SARS-COV-2: An integrative review \\ Perfil clínico, prevalencia y mortalidad de pacientes hospitalizados con enfermedades hepáticas \\ crónicas infectadas por SARS-COV-2: Una revisión integradora
}

Recebido: 20/02/2021 | Revisado: 28/03/2021 | Aceito: 01/04/2021 | Publicado: 12/04/2021

Thyago de Oliveira Afonso ORCID: https://orcid.org/0000-0001-7616-9011 Universidade Federal de Pernambuco, Brasil

E-mail: thyago.oafonso@gmail.com

Sarah Maria Soares de Freitas ORCID: https://orcid.org/0000-0002-3912-046X Universidade Federal de Pernambuco, Brasil

E-mail: sarahmsdefreitas@gmail.com

Douglas Rogério Freitas de Souza

ORCID: https://orcid.org/0000-0002-4300-156X

Universidade Federal de Pernambuco, Brasil

E-mail: mailto:douglas.rogerio@ufpe.br

Gabriel Vítor Lima de Andrade

ORCID: https://orcid.org/0000-0001-6057-8486

Universidade Federal de Pernambuco, Brasil

E-mail:gabriel.limaandrade@ufpe.br

José Erivaldo Gonçalves

ORCID: http://orcid.org/0000-0001-7841-8296 Instituto Aggeu Magalhães IAM/FIOCRUZ PE, Brasil

E-mail: goncalves.erij@gmail.com

Mariana Pereira Barbosa Silva

ORCID: https://orcid.org/0000-0003-0852-8099

Universidade Estadual do Piauí, Brasil

E-mail: marianapbsilvaa@gmail.com

Samuel Lopes dos Santos

ORCID: https://orcid.org/0000-0003-3375-9171 Universidade Federal do Piauí, Brasil

E-mail: samuellopes121314@gmail.com

Simone Barroso de Carvalho

ORCID: https://orcid.org/0000-0001-7428-8420 Universidade Federal do Piauí, Brasil

E-mail: simonebcarvalho2009@hotmail.com

Leandro Luiz da Silva Loures

ORCID: https://orcid.org/0000-0001-8863-8858

Universidade Federal de Juiz de Fora, Brasil

E-mail: leandrolouresnutri@gmail.com

Gustavo Baroni Araujo

ORCID: https://orcid.org/0000-0002-3162-7477

Universidade Estadual de Londrina, Brasil

E-mail: gustavobaroni13@hotmail.com

Beatriz Cardoso Melo

ORCID: https://orcid.org/0000-0002-3638-8815

Centro Universitário Uninovafapi, Brasil

E-mail: beatrizcmelo@outlook.com

Yulle Morais Gomes

ORCID: https://orcid.org/0000-0003-0715-2106

Centro Universitário UniFacid Wyden, Brasil

E-mail: yullemorais100@hotmail.com

Luís Eduardo Arcoverde Nogueira Brayner ORCID: https://orcid.org/0000-0002-1702-3319

Centro Universitário UniFacid Wyden, Brasil

E-mail: luiseduardobrayner92@gmail.com 


\author{
Natana Maranhão Noleto da Fonseca \\ ORCID: https://orcid.org/0000-0003-4592-3004 \\ Centro Universitário Uninovafapi, Brasil \\ E-mail: natana_fonseca1@hotmail.com \\ Maria Clara Osório Meneses Carvalho \\ ORCID: https://orcid.org/0000-0003-2603-5159 \\ Centro Universitário UniFacid Wyden, Brasil \\ E-mail: mcosorio97@yahoo.com.br \\ Mariana Magalhães Bergantini Zanovello \\ ORCID: https://orcid.org/0000-0002-2932-9219 \\ Centro Universitário UniFacid Wyden, Brasil \\ E-mail: mariana_zanovello@hotmail.com \\ Ilana Marjorie Macedo Borges Miranda \\ ORCID: https://orcid.org/0000-0002-3576-6474 \\ Centro Universitário Uninovafapi, Brasil \\ E-mail: ilana_miranda10@hotmail.com \\ Amanda Viana Santos \\ ORCID: https://orcid.org/0000-0002-4520-0634 \\ Centro Universitário Tiradentes, Brasil \\ E-mail: amandavianas@hotmail.com \\ Amanda Costa Maciel \\ ORCID: https://orcid.org/0000-0002-2668-037X \\ Faculdade São Vicente de Pão de Açúcar, Brasil \\ E-mail: amandacmaciel@hotmail.com \\ Yonara Cristiane Ribeiro \\ ORCID: https://orcid.org/0000-0002-6868-1629 \\ Universidade Federal Fluminense, Brasil \\ E-mail: yonaracristiane@id.uff.br \\ Pâmela Ferreira Brito \\ ORCID: https://orcid.org/0000-0001-6780-4116 \\ Centro Universitário de Belo Horizonte, Brasil \\ E-mail: pamelafbrito@hotmail.com
}

\begin{abstract}
Resumo
Objetivo: Analisar evidências científicas sobre perfil clínico, prevalência e mortalidade de pacientes adultos hospitalizados com doença hepática crônica (DHC) infectados com SARS-COV-2. Metodologia: Trata-se de uma revisão bibliográfica no método revisão integrativa de literatura, realizado em fevereiro de 2021, nas bases de dados MEDLINE/PubMed, EMBASE e LILACS, por meio dos descritores/MESH: "COVID-19" AND "chronic liver disease", combinados com o operador booleano "AND". Foram critérios de inclusão: artigos originais disponíveis na íntegra, completos, publicados entre 2019 e janeiro de 2021, nos idiomas português, inglês e/ou espanhol, e temática condizente. Os critérios de exclusão foram: artigos incompletos, duplicados, sem relação com o tema, em pre-proof, teses, dissertações, monografias e manuais. Resultados: Foram incluídos sete artigos. Foram analisados 1693 pacientes adultos hospitalizados infectados com SARS-COV-2 e obteve-se frequência variando entre $0,79 \%$ e $50 \%$, em comparação a população hospitalizada infectada com COVID-19 com e sem DHC. O perfil clínico dos pacientes com DHC e COVID-19 teve predominância masculina, média de idade: 55 anos, doença hepática gordurosa não alcoólica como DHC prévia e hipertensão como comorbidade principal. Os sintomas mais relatados foram tosse $(80,8 \%)$, presença de infecção(73,1\%), expectoração(26,9\%), dispneia(25\%), mialgia ou fadiga(13,5\%). As principais alterações laboratoriais e de imagem incluíram elevação nas enzimas hepáticas, linfopenia, opacidades difusas e padrão de vidro fosco. A mortalidade foi mais significativa em pacientes DHC e COVID-19 imunossuprimidos variando entre $1,78 \%$ e $8,1 \%$. Conclusão: Os dados propõem uma tendência de perfil clínico, prevalência e mortalidade, porém há necessidade de outros estudos coorte ou ensaios clínicos randomizados com amostras maiores para confirmar hipóteses.
\end{abstract}

Palavras-chave: Doença hepática crônica; Infecção por coronavírus; Coinfecção; Mortalidade.

\begin{abstract}
Objective: To analyze scientific evidence on the clinical profile, prevalence and mortality of adult hospitalized patients with chronic liver disease (CLD) infected with SARS-COV-2. Methodology: This is a bibliographic review in the integrative literature review method, carried out in February 2021, in the databases MEDLINE / PubMed, EMBASE and LILACS, using the descriptors / MESH: "COVID-19" AND "chronic liver disease ", combined with the Boolean operator "AND". Inclusion criteria were: original articles available in full, complete, published between 2019 and January 2021, in Portuguese, English and/or Spanish, and a suitable theme. The exclusion criteria were: incomplete, duplicate articles, unrelated to the topic, in pre-proof, theses, dissertations, monographs and manuals. Results: Seven articles were included. 1693 adult hospitalized patients infected with SARS-COV-2 were analyzed and the frequency was varied between $0.79 \%$ and $50 \%$, compared to the hospitalized population infected with COVID-19 with and without CLD. The clinical profile of patients with CLD and COVID-19 was predominantly male, mean age:
\end{abstract}


55 years, non-alcoholic fatty liver disease as a previous CLD and hypertension as the main comorbidity. The most reported symptoms were cough $(80.8 \%)$, presence of infection(73.1\%), sputum(26.9\%), dyspnea(25\%), myalgia or fatigue(13.5\%). The main laboratory and imaging changes included elevation in liver enzymes, lymphopenia, diffuse opacities and ground-glass. Mortality was more significant in immunosuppressed CLD and COVID-19 patients, ranging from $1.78 \%$ to $8.1 \%$. Conclusion: The data propose a trend in clinical profile, prevalence and mortality, but there is a need for further cohort studies or randomized clinical trials with larger samples to confirm hypotheses.

Keywords: Chronic liver disease; Coronavirus infection; Coinfection; Mortality.

\begin{abstract}
Resumen
Objetivo: Analizar la evidencia científica sobre el perfil clínico, prevalencia y mortalidad de pacientes adultos hospitalizados con enfermedad hepática crónica (EHC) infectados con SARS-COV-2. Metodología: Se trata de una revisión bibliográfica en el método de revisión integrativa de la literatura, realizada en febrero de 2021, en las bases de datos MEDLINE/PubMed, EMBASE y LILACS, utilizando los descriptores/MESH: "COVID-19" Y "enfermedad hepática crónica", combinados con el operador booleano "Y". Los criterios de inclusión fueron: artículos originales disponibles en su totalidad, completos, publicados entre 2019 y enero de 2021, en portugués, inglés y/o español, y temática coherente. Los criterios de exclusión fueron: artículos incompletos, duplicados, ajenos al tema, en preprueba, tesis, disertaciones, monografías y manuales. Resultados: Se incluyeron siete artículos. Se analizaron 1693 pacientes adultos hospitalizados infectados por SARS-COV-2 y la frecuencia varió entre $0,79 \%$ y $50 \%$, en comparación con la población hospitalizada infectada por COVID-19 con y sin EHC. El perfil clínico de los pacientes con EHC y COVID-19 fue predominantemente masculino, edad media: 55 años, hígado graso no alcohólico como EHC previo e hipertensión como principal comorbilidad. Los síntomas más reportados fueron tos $(80,8 \%)$, presencia de infección( $73,1 \%)$, esputo(26,9\%), disnea( $25 \%)$, mialgia o fatiga(13,5\%). Los principales cambios de laboratorio y de imagen incluyeron elevación de las enzimas hepáticas, linfopenia, opacidades difusas y patrón de vidrio esmerilado. La mortalidad fue más significativa en los pacientes inmunosuprimidos con EHC y COVID-19, con un rango de $1,78 \%$ a $8,1 \%$. Conclusión: Los datos proponen una tendencia en el perfil clínico, la prevalencia y la mortalidad, pero se necesitan más estudios de cohortes o ensayos clínicos aleatorizados con muestras más grandes para confirmar las hipótesis.
\end{abstract}

Palabras clave: Enfermidad hepática crónica; Infecciones por Coronavirus; Coinfección; Mortalidad.

\title{
1. Introdução
}

A atual doença causada pelo coronavírus de 2019 (COVID-19), causada pelo coronavírus da síndrome respiratória aguda grave (SARS-COV-2), tem sido uma crise desafiadora para os profissionais de saúde, cientistas, epidemiologistas, governantes, economistas e principalmente para a saúde pública (Stasi et al., 2020). Devido ao panorama de mortalidade e expansão do vírus, a Organização Mundial da Saúde (OMS) declarou emergência de saúde pública internacional a partir de 30 de janeiro de 2020 (Gautret et al., 2020).

A maioria dos casos de infecção pelo SARS-COV-2 são benignos, autolimitados e sem fatalidades. Contudo formas severas e críticas da COVID-19 apresentam-se em aproximadamente $20 \%$ dos pacientes infectados, necessitando de ventilação mecânica e cuidados de terapia intensiva. Segundo a OMS os fatores de risco para pior prognóstico na COVID-19 são: populações idosas ou em envelhecimento, doenças renais crônicas, doença pulmonar obstrutiva crônica (DPOC), obesidade, imunocomprometido, transplante de órgão sólido, condições cardíacas graves, anemia falciforme, diabetes tipo 2, asma, hipertensão, condições neurológicas e gestação (Gautret et al., 2020). De acordo com Stasi et al (2020) pode afetar principalmente os pulmões, coração, fígado, pâncreas, rins e o intestino.

No que se trata de coinfecção da COVID-19 em pacientes com hepatites crônicas, entende-se como qualquer alteração na função hepática clínica ou analítica desses pacientes com patologia pré-existentes e sãos. As doenças hepáticas crônicas (DHC) pré-existentes são multifatoriais, contudo, as mais importantes são de diagnóstico de cirrose, vírus da hepatite B (HBV), vírus da hepatite $\mathrm{C}(\mathrm{HCV})$, doença hepática alcoólica (DHA), doença hepática gordurosa não alcoólica (DHGNA), sendo a mais prevalente no mundo, ou carcinoma hepatocelular (CHC) (Jothimani et al., 2020).

Em um estudo prévio foi identificado que pacientes com DHC coinfectados com COVID-19 apresentaram incidência entre 0,6 e 1,4\% dos pacientes hospitalizados com COVID-19, dentre os quais $60 \%$ tiveram apresentação grave da doença e 
com uma mortalidade de aproximadamente $18 \%$, tendo a apresentação mais comum associada a doença hepática gordurosa não alcoólica (Gómez et al., 2020).

Tendo em vista este quadro de infecção, as alterações no perfil clínico dos pacientes hospitalizados com doença hepática crônica e COVID-19 são esperados, assim como a incidência de agravos na condição pré-existente pela cascata inflamatória da COVID-19 (Gómez et al., 2020). Diante disto, o objetivo deste estudo foi investigar o perfil clínico, prevalência e mortalidade destes pacientes crônicos hospitalizados com infecção com o SARS-COV-2.

\section{Metodologia}

Trata-se de uma estudo de revisão bibliográfica e exploratório no método revisão integrativa de literatura, realizado entre os meses de janeiro e 19 de fevereiro de 2021, com abordagem descritiva, nas bases de dados do Sistema Online de Busca e Análise de Literatura Médica (Medical Literature Analysis and Retrieval System Online) da Biblioteca Nacional de Medicina dos Estados Unidos da América (US National Library of Medicine - NLM) - MEDLINE/PubMed, Banco de Dados Bibliográficos Biomédico e Farmacológico (Excerpta Medica dataBASE) - EMBASE e Literatura Latino-americana e do Caribe em Ciências da Saúde - LILACS, através dos descritores/ palavras-chave: "COVID-19" AND "chronic liver disease", combinados com o operador booleano "AND".

Tratando-se de uma pesquisa exploratória e descritiva dos dados, assumindo uma gama de conhecimento, fenômenos e problemas, com o objetivo principal de descrever e quantificar a natureza das variáveis (Koche, 2011).

Para execução deste estudo, foram seguidas as etapas de pesquisa: 1- Elaboração de pergunta norteadora; 2- Busca ou amostragem na literatura; 3- Coleta de dados; 4- Análise crítica dos estudos incluídos; 5- Discussão dos resultados; 6Apresentação dos resultados em revisão integrativa (Koche, 2011; Pereira et al., 2018).

Para responder à pergunta norteadora: "Qual o perfil clínico e epidemiológico, prevalência e mortalidade de pacientes adultos hospitalizados com doenças hepáticas crônicas infectados com SARS-COV-2?", foi realizada busca de artigos nas bases de dados da MEDLINE/Pubmed, EMBASE e Literatura Latino-Americana e do Caribe em Ciências da Saúde (LILACS), com os descritores/MESH: "COVID-19" AND "chronic liver disease", utilizando o operador booleano AND. Os critérios de inclusão foram artigos originais disponíveis na íntegra, completos, publicados entre 2019 e janeiro de 2021, identificados entre 10 de janeiro e 19 de fevereiro de 2021, nos idiomas português, inglês e/ou espanhol, e temática condizente. Foram excluídos artigos incompletos, cartas, editoriais, duplicados, sem relação com o tema, em pre-proof, teses, dissertações, monografias e manuais.

Após a seleção, foram avaliados os resumos e títulos a fim de identificar a temática condizente para este estudo, através de avaliação em pares por oito estudantes e em caso de divergências, analisado por dois outros estudantes. Nos casos em que a leitura não foi suficiente para definir a inclusão do artigo na amostra, foram considerados os demais critérios. A elegibilidade foi determinada pela leitura na íntegra dos artigos selecionados. Por fim, os dados referentes ao fluxograma do processo de identificação e seleção dos artigos estão sistematicamente demonstrados na Figura 01, elaborada pelos pesquisadores.

\section{Resultados}

A busca inicial nas bases de dados a partir dos descritores/MESH retornou 1.074 trabalhos, em sua maior parte obtida do EMBASE ( $\mathrm{n}=674)$, seguido de MEDLINE/Pubmed $(\mathrm{n}=371)$ e LILACS $(\mathrm{n}=7)$. Deste total, após aplicados os critérios de inclusão e exclusão foram selecionados 45 trabalhos para leitura na íntegra, entretanto, após aplicados os critérios de elegibilidade, apenas sete foram aprovados para este estudo, como esquematizado na Figura 1. 
Figura 1: Fluxograma de processo de identificação e seleção de artigos.
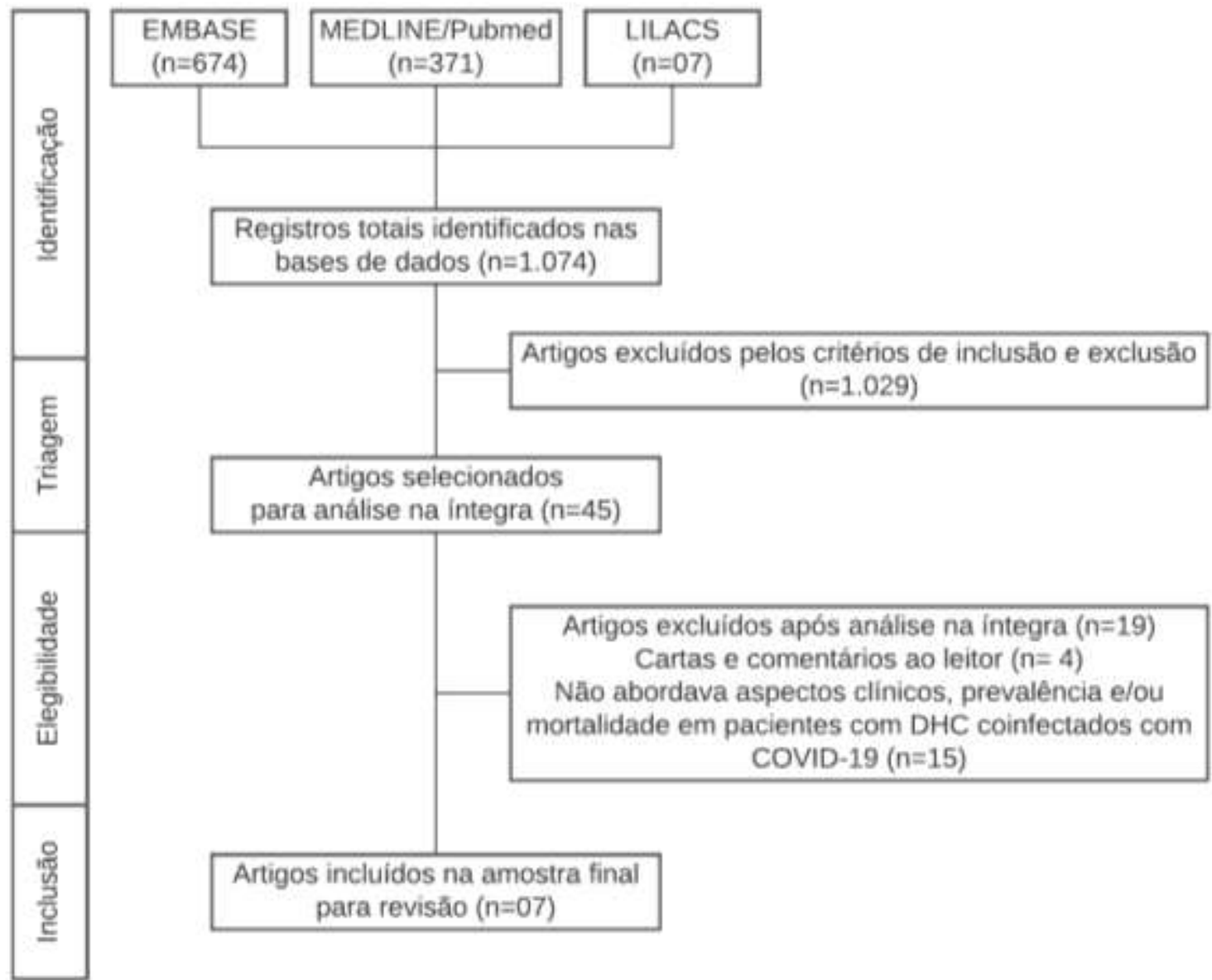

Artigos incluidos na amostra final para revisăo $(n=07)$

Fonte: Autores.

Foram selecionados sete artigos, sendo um artigo de série de casos controle, dois artigos de série de caso retrospectivo, dois artigos de estudo de coorte retrospectivos e dois artigos de meta-análises, conforme demonstrado no Quadro 1. Os artigos foram realizados em centros diferentes e produzidos durante o ano de 2020. Quanto ao idioma, todos os artigos foram publicados na língua inglesa.

Quadro 1: Caracterização dos estudos incluídos na revisão integrativa segundo autores/ano da publicação, título, desenho do estudo, local do estudo e ano da pesquisa.

\begin{tabular}{|c|c|c|c|}
\hline $\begin{array}{l}\text { Autor/Ano da } \\
\text { Publicação }\end{array}$ & Título & Desenho do Estudo & País/Ano da Pesquisa \\
\hline JI et al., 2020 & $\begin{array}{l}\text { Effect of COVID- } 19 \text { on patients with } \\
\text { compensated chronic liver diseases. }\end{array}$ & $\begin{array}{l}\text { Estudo de coorte } \\
\text { retrospectivo }\end{array}$ & China/2020 \\
\hline WANG et al., 2020 & $\begin{array}{l}\text { COVID-19 risk, disparities and outcomes in } \\
\text { patients with chronic liver disease in the United } \\
\text { States. }\end{array}$ & Estudo de caso controle & $\begin{array}{l}\text { Estados Unidos da } \\
\text { América/ } 2020\end{array}$ \\
\hline $\begin{array}{l}\text { RICHARDSON et } \\
\text { al., } 2020\end{array}$ & $\begin{array}{l}\text { Characteristics, Comorbidities, and Outcomes } \\
\text { Among } 5700 \text { Patients Hospitalized With } \\
\text { COVID-19 in the New York City Area }\end{array}$ & Série de casos retrospectiva & $\begin{array}{l}\text { Estados Unidos da } \\
\text { América/2020 }\end{array}$ \\
\hline $\begin{array}{l}\text { KOVALIC ET AL., } \\
2020\end{array}$ & $\begin{array}{l}\text { Prevalence of chronic liver disease in patients } \\
\text { with COVID-19 and their clinical outcomes: a } \\
\text { systematic review and meta-analysis }\end{array}$ & Meta-análise & Multicêntrico/2020 \\
\hline
\end{tabular}




\begin{tabular}{|c|c|c|c|}
\hline LI ET AL., 2020 & $\begin{array}{l}\text { Clinical characteristics of chronic liver disease } \\
\text { with coronavirus disease } 2019 \text { (COVID-19): a } \\
\text { cohort study in Wuhan, China }\end{array}$ & $\begin{array}{l}\text { Estudo de coorte } \\
\text { retrospectivo }\end{array}$ & China/2020 \\
\hline $\begin{array}{l}\text { MANTOVANI ET } \\
\text { AL., } 2020\end{array}$ & $\begin{array}{l}\text { Coronavirus disease } 2019 \text { and prevalence of } \\
\text { chronic liver disease: A meta-analysis }\end{array}$ & Meta-análise & Multicêntrico/2020 \\
\hline $\begin{array}{l}\text { GRASELLI ET AL., } \\
\qquad 2020\end{array}$ & $\begin{array}{l}\text { Baseline Characteristics and Outcomes of } 1591 \\
\text { Patients Infected With SARS-CoV-2 Admitted } \\
\text { to ICUs of the Lombardy Region, Italy }\end{array}$ & Série de casos retrospectiva & Itália/2020 \\
\hline
\end{tabular}

Fonte: Autores.

Os principais resultados dos estudos selecionados foram sintetizados no Quadro 02, avaliando os principais resultados por número de pacientes com DHC hospitalizados infectados com SARS-COV-2, prevalência em relação a população hospitalizada infectada com COVID-19, gênero, idade, apresentação de DHC, comorbidades associadas, perfil clínico e exames laboratoriais, exames de imagem, evolução e mortalidade dos pacientes com doença hepática crônica infectados com COVID-19. Foram avaliados 1.693 pacientes adultos hospitalizados com doenças hepáticas crônicas (DHC) infectados com SARS-COV-2. Todos os artigos utilizaram o exame laboratorial do RT-PCR para identificação do SARS-COV-2 em amostra de fluído nasofaríngeo e confirmação de COVID-19.

A prevalência destes pacientes em relação a pacientes hospitalizados sem DHC obteve uma média variável entre 0,79\% a 50\%, com maior acometimento em população masculina, com idade acima de 18 e média de 55 anos. As apresentações mais comuns da DHC nos pacientes com DHC e infectados com SARS-COV-2 foram a cirrose hepática alcoólica, doença hepática gordurosa não alcoólica, hepatites virais do tipo $\mathrm{B}$ e $\mathrm{C}$, transplante de fígado e dano hepático alcoólico.

Os sinais e sintomas mais comuns relatados foram: tosse $(80,8 \%)$, presença de infecção $(73,1 \%)$, expectoração $(26,9 \%)$, dispnéia $(25 \%)$ e mialgia ou fadiga (13,5\%). Dentre os achados laboratoriais, destaca-se a alteração das enzimas hepáticas, linfopenia, velocidade de hemossedimentação aumentada, níveis aumentados de bilirrubina total e aumento dos marcadores de inflamação. Dentre os exames de imagem, os mais utilizados foram: radiografia de tórax e tomografia computadorizada, as quais localizaram em sua maioria opacificações difusas e padrão de vidro fosco.

As comorbidades mais presentes nos pacientes com DHC infectados com SARS-COV-2 foram hipertensão arterial sistêmica, diabetes, obesidade, asma e doenças mentais prévias. Com relação ao manejo clínico, este varia através das apresentações e criticidade dos pacientes, mas em geral, a apresentação mais grave necessitou de tratamento em Unidade de Terapia Intensiva (UTI), oxigenoterapia, corticoterapia, intubação orotraqueal para ventilação mecânica invasiva e antibioticoterapia.

A mortalidade nesta população apresentou variação média entre $1,78 \%$ a 8,1\% para pacientes maiores de 18 anos. Cabe ressaltar que, segundo Wang e colaboradores (2020), para pacientes afro-americanos com DHC infectados com SARSCOV-2 a mortalidade apresentou-se $2 x$ maior que em população caucasiana nos Estados Unidos da América. 


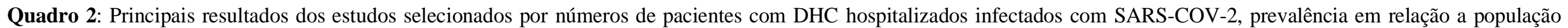

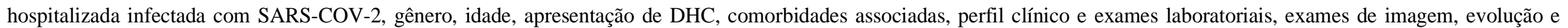
mortalidade dos pacientes com doença hepática crônica hospitalizados infectados com SARS-COV-2

\begin{tabular}{|c|c|c|c|c|c|c|c|}
\hline Autores & JI et al., 2020 & WANG et al., 2020 & RICHARDSON et al., 2020 & KOVALIC et al., 2020 & LI et al., 2020 & $\begin{array}{l}\text { MANTOVANI et al., } \\
2020\end{array}$ & GRASELLI et al., 2020 \\
\hline $\begin{array}{l}\text { Número de pacientes } \\
\text { hospitalizados com DHC } \\
\text { infectados com SARS- } \\
\text { COV-2 }\end{array}$ & 22 pacientes & 820 pacientes & 60 pacientes & 650 pacientes & 52 pacientes & 62 pacientes & 27 pacientes \\
\hline $\begin{array}{l}\text { Prevalência em relação a } \\
\text { população hospitalizada } \\
\text { infectada com SARS- } \\
\text { COV-2 }\end{array}$ & $15,7 \%$ & $4,96 \%$ & $0,79 \%$ & $18,20 \%$ & $50 \%$ & $3 \%$ & $2,58 \%$ \\
\hline Gênero & $\begin{array}{l}14 \text { masculinos } \\
(63,6 \%) \text { e } 8 \\
\text { femininos }(36,4 \%)\end{array}$ & $\begin{array}{l}450 \text { masculinos } \\
(55 \%) \text { e } 370 \\
\text { femininos }(45 \%)\end{array}$ & $\begin{array}{l}60,3 \% \text { masculinos e } 39,7 \% \\
\text { femininos }\end{array}$ & Não especificado & $\begin{array}{l}32 \text { masculinos }(61,5 \%) \text { e } 20 \\
\text { femininos }(38,5 \%)\end{array}$ & Não especificado & $\begin{array}{l}22 \text { masculinos }(81,5 \%) \text { e } 5 \\
\text { femininos }(18,5 \%)\end{array}$ \\
\hline Idade & $53,0 \pm 14,5$ anos & $\begin{array}{l}580 \text { adultos }(18-65 \\
\text { anos) e } 240 \text { idosos } \\
(>65 \text { anos) }\end{array}$ & $63,0 \pm 09,0$ anos & Não especificado & $58.2 \pm 11.7$ & 49 anos em média & $563,0 \pm 07,0$ \\
\hline Apresentação de DHC & $\begin{array}{l}3(13,6 \%) \text { cirrose } \\
\text { alcoólica, } 6 \\
(27,2 \%) \text { hepatite B } \\
\text { crônica, } 13 \\
(59,1 \%) \text { doença } \\
\text { hepática gordurosa } \\
\text { não alcoólica }\end{array}$ & $\begin{array}{l}50(6,09 \%) \text { cirrose } \\
\text { alcoólica, } 150 \\
(18,29 \%) \text { cirrose não } \\
\text { alcoólica, } 570 \\
(69,51 \%) \text { doença } \\
\text { hepática gordurosa } \\
\text { não alcoólica, } 180 \\
(21,95 \%) \text { hepatite C } \\
\text { crônica, } 30(3,65 \%) \\
\text { hepatite B crônica, } 70 \\
(8,53 \%) \text { dano } \\
\text { hepático alcoólico }\end{array}$ & Não especificado & $\begin{array}{l}\text { Cirrose, vírus da hepatite } \\
\text { B, vírus da hepatite C, } \\
\text { Doença Hepática } \\
\text { Gordurosa Não Alcoólica }\end{array}$ & Não especificado & $\begin{array}{l}\text { Infecção pelo vírus da } \\
\text { hepatite C, vírus da } \\
\text { hepatite B com } \\
\text { prevalência na forma } \\
\text { crônica. }\end{array}$ & $\begin{array}{l}\text { Principalmente relacionado a } \\
\text { hepatite B crônica. }\end{array}$ \\
\hline Comorbidades associadas & $\begin{array}{l}13(59,1 \%) \\
\text { diabéticos. }\end{array}$ & Não especificado & $\begin{array}{l}\text { hipertensão }(56,6 \%) \text {, diabetes } \\
(33,8 \%) \text { e obesidade }(41,7 \%)\end{array}$ & $\begin{array}{l}\text { hipertensão, diabetes, } \\
\text { obesidade, asma, doenças } \\
\text { mentais. }\end{array}$ & $\begin{array}{l}38 \% \text { dos pacientes } \\
\text { apresentavam comorbidades. }\end{array}$ & Não especificado. & $\begin{array}{l}\text { Pelo menos } 49 \% \text { dos } \\
\text { pacientes apresentaram uma } \\
\text { comorbidade (hipertensão). }\end{array}$ \\
\hline $\begin{array}{l}\text { Perfil clínico e Exames } \\
\text { laboratoriais }\end{array}$ & $\begin{array}{l}\text { RT-PCR de swab } \\
\text { nasofaríngeo } \\
\text { positivo para } \\
\text { SARS-COV-2. } \\
\text { Elevação sérica de } \\
\text { ALT, AST, TBIL, } \\
\text { alto nível de IL-6, } \\
\text { TNF-alfa, ferritina } \\
\text { e INR em }\end{array}$ & Não especificado & $\begin{array}{l}\text { RT-PCR de swab nasofaríngeo } \\
\text { positivo para SARS-COV-2. } \\
\text { Elevação sérica de ALT ou } \\
\text { ALP, AST, TBIL, alto nível de } \\
\text { IL-6, TNF-alfa, ferritina e INR } \\
\text { em pacientes com cirrose. }\end{array}$ & $\begin{array}{l}\text { RT-PCR swab } \\
\text { nasofaríngeo positivo para } \\
\text { SARS-COV-2. Alteração } \\
\text { de enzimas hepáticas } \\
\text { como prognóstico para } \\
\text { pior evolução. }\end{array}$ & $\begin{array}{l}\text { Os sintomas iniciais } \\
\text { apresentados foram: dispnéia } \\
(25 \%) \text {, tosse }(80,8 \%), \\
\text { expectoração }(26,9 \%), \\
\text { mialgia ou fadiga }(13,5 \%) \text {, } \\
\text { frequência respiratória } \\
\text { média de } 22 \text { irp, presença de } \\
\text { infeção }(73,1 \%) \text {. Os } \\
\text { exames laboratoriais }\end{array}$ & $\begin{array}{l}\text { Os níveis de AST sérico } \\
\text { variaram entre } 26 \text { UI/L e } \\
46 \text { UI/L, com ALT sérico } \\
\text { variando entre } 22 \text { UI/L e } \\
41 \text { UI/L. Os tempos de } \\
\text { protrombina e níveis de } \\
\text { bilirrubinas totais } \\
\text { aumentaram. RT-PCR } \\
\text { swab nasofaríngeo }\end{array}$ & $\begin{array}{l}\text { RT-PCR swab nasofaríngeo } \\
\text { positivo para SARS-COV-2. }\end{array}$ \\
\hline
\end{tabular}


Research, Society and Development, v. 10, n. 4, e33010413143, 2021

(CC BY 4.0) | ISSN 2525-3409 | DOI: http://dx.doi.org/10.33448/rsd-v10i4.13143

pacientes com

cirrose.

\begin{tabular}{|c|c|c|c|c|}
\hline Exames de imagem & $\begin{array}{l}\text { Radiografia de } \\
\text { tórax com } \\
\text { múltiplas } \\
\text { opacidades em } \\
\text { padrão de vidro } \\
\text { fosco em ambos os } \\
\text { pulmões }\end{array}$ & Não especificado & $\begin{array}{l}\text { Radiografia de tórax e/ou } \\
\text { tomografia computadorizada } \\
\text { com múltiplas opacidades em } \\
\text { padrão de vidro fosco }\end{array}$ & $\begin{array}{l}\text { Tomografia } \\
\text { computadorizada com } \\
\text { múltiplas opacidades e } \\
\text { necrose tecidual. }\end{array}$ \\
\hline Evolução e manejo & $\begin{array}{l}\text { Oxigenoterapia, } \\
\text { Ventilação } \\
\text { mecânica, } \\
\text { metilprednisolona,i } \\
\text { munoglobulinas, }\end{array}$ & $\begin{array}{l}41 \% \text { de taxa de } \\
\text { hospitalização. }\end{array}$ & $\begin{array}{l}\text { Tratamento em Unidade de } \\
\text { Tratamento Intensivo }(12,2 \%) \text {, } \\
\text { Intubação Orotraqueal para } \\
\text { ventilação }(12,2 \%) .\end{array}$ & $\begin{array}{l}\text { Tratamento em Unidade } \\
\text { de Tratamento Intensivo } \\
(27,9 \%)\end{array}$ \\
\hline
\end{tabular}

Mortalidade dos pacientes

hospitalizados com DHC

infectados com SAR

1/21 (um paciente

hepática crônica

Afro-americanos com $\quad 8,1 \%$ em pacientes com idade

maior que 65 anos.

SARS-COV-2

possuem $2 \mathrm{x}$ mais

risco de morte que

caucasianos. registram elevação na razão

IL-6, AST, ALT e

plaquetopenia. RT-PCR

swab nasofaríngeo positivo

para SARS-COV-2.

Tomografia

computadorizada de tórax

com apresentação de vidro

fosco unilateral $(13,5 \%)$

bilateral $(86,5 \%)$.

Antibioticoterapia (78,8\%),

corticoterapia $(30,8 \%)$

suporte de oxigênio $(76,9 \%)$.

Evolução relacionada a

razão neutrófilo/linfócito

como índice para pior

prognóstico.
Relacionada ao processo
mais severo da COVID-19
alinhado a cirrose $\mathrm{e}$

transplante de fígado par

pacientes com DHC

pacientes
$(1,78 \%)$

9/52 pacientes $(17,3 \%)$ positivo para SARS-COV-

Não especificado

Tomografia computadorizada de tórax com apresentação de vidro fosco

Os altos níveis de enzimas

hepáticas foram

Ventilação mecânica

marcadores para pior

para os pacientes,

mostrando alta ativação

coagulação e processo

fibrinolíticos. O manejo

através da investigação da

causa à injúria hepática

tratamento direcionado

para COVID-19 lesionar o

fígado.

Não especificado.

15-30\% de mortalidade em

Legenda: RT-PCR: Reverse-transcriptase polymerase chain reaction; ALT: alanina aminotransferase; AST: aspartato aminotransferase; TBIL: bilirrubina total; IL-6: interleucina 06; TNF-alfa: fator de necrose tumoral alfa; INR: razão de normatização internacional; CK: creatinoquinase; PEEP: pressão expiratória final positiva.

Fonte: Autores. 
Research, Society and Development, v. 10, n. 4, e33010413143, 2021

(CC BY 4.0) | ISSN 2525-3409 | DOI: http://dx.doi.org/10.33448/rsd-v10i4.13143

\section{Discussão}

A infecção causada pelo coronavírus, denominado SARS-COV-2, é chamada de doença do coronavírus 2019 (COVID-19), que surgiu na China no final de 2019 (Stasi et al., 2020). Até o dia 19 de fevereiro de 2021, já haviam sido registrados mais de 110 milhões de casos de infecção pelo coronavírus causador da síndrome respiratória aguda grave 2 (SARS-COV-2), com cerca de 2,4 milhões de óbitos.

A mortalidade na população geral infectada com COVID-19 gira em torno de 2 a 6\%, entretanto outros fatores de risco, como diabetes, hipertensão, doença cardíaca e outros fatores imunossupressores podem predispor o paciente a formas mais severas da doença (Stasi et al., 2020).

Diante disso, é observado que pacientes com doenças hepáticas crônicas, como cirrose, doença hepática gordurosa não alcoólica e outras, podem tornar a função imune irresponsiva ou debilitada, e assim, caso haja infecção ela SARS-COV-2 pode desenvolver formas mais agravadas da COVID-19 (Jothimani et al., 2020; Moon et al., 2019).

Neste contexto pandêmico, estabelecer os perfis clínicos, prevalência e mortalidade dos pacientes hospitalizados com DHC infectados com SARS-COV-2 se torna essencial para projetar medidas futuras em saúde pública para esta população.

Um dos pontos importantes para o perfil clínico dos pacientes hospitalizados com DHC infectados com SARS-COV-2 foi observado que nesta população o sexo masculino teve uma frequência de (71,42\%), que confirmado por Teich et al. (2020), aponta também maior frequência de incidência de infecção pelo SARS-COV-2 no sexo masculino, além de já haver pesquisas confirmando esse mesmo perfil para pacientes com DHC sem infecção por SARS-COV-2 (Moon et al., 2019).

No que tange à idade, a média dos 1.693 pacientes adultos hospitalizados com DHC infectados com SARS-COV-2 avaliados neste estudo obtiveram uma média de 55 anos, média superior à observada em pacientes infectados com COVID-19 isoladamente que é de 39,9 anos (Teich et al., 2020). Quando analisada a correlação entre a idade em pacientes com DHC e a gravidade da COVID-19, Rentsch et al. (2020) e Xiao et al., (2020) apontam que a idade pode ser fator determinante para pior prognóstico, assim como a correlação com obesidade e o sexo masculino.

Segundo Jothimani et al. (2020), as patologias mais prevalentes que mais se apresentaram em pacientes hospitalizados com DHC infecção com SARS-COV-2 foram as doenças hepáticas gordurosas não alcoólicas, hepatites virais do tipo B e C, e cirrose, dada a descompensação hepática ocasionada pela cascata de citocinas inflamatórias devida a patologia prévia e a COVID-19, aumentado a mortalidade por consequência. Nosso estudo reforça esta afirmação apresentando dados quantitativos sobre a apresentação de doença hepática gordurosa não alcoólica com apresentação em 883 (52,46\%) pacientes, sendo a patologia mais frequente, seguida de hepatite $\mathrm{C}$, hepatite $\mathrm{B}$ e cirrose alcoólica.

No presente estudo, as principais comorbidades associadas analisadas foram hipertensão, diabetes, obesidade, asma e doenças mentais, e verifica-se que não há dados quantitativos suficientes para realizarmos uma análise mais aprofundada (Jothimani et al., 2020). Contudo, Barry et al. (2020) correlaciona a descompensação hepática a achados neoplásicos no manejo inicial da COVID-19 nestes pacientes, o que favorece um descontrole do sistema imune hospedeiro, resultando num agravamento pelo quadro neutropênico e séptico do paciente.

Os sinais e sintomas mais comuns apresentados pelos adultos hospitalizados com DHC infectados com SARS-COV-2 entre os pacientes hospitalizados com COVID-19 foram tosse (80,8\%), presença de infecção (73,1\%), expectoração (26,9\%), dispneia $(25 \%)$ e mialgia ou fadiga $(13,5 \%)$. Dentre os achados laboratoriais, destaca-se a alteração das enzimas hepáticas, linfopenia, velocidade de hemossedimentação aumentada, níveis aumentados de bilirrubina total e aumento dos marcadores de inflamação. Dentre os exames de imagem, os mais utilizados foram: radiografia de tórax e tomografia computadorizada, as quais localizaram em sua maioria opacificações difusas e padrão de vidro fosco. Estes achados de perfil sintomatológico, 
Research, Society and Development, v. 10, n. 4, e33010413143, 2021

(CC BY 4.0) | ISSN 2525-3409 | DOI: http://dx.doi.org/10.33448/rsd-v10i4.13143

clínico, laboratorial e de imagem são previstos pela Diretrizes para Diagnóstico e Tratamento da COVID-19 (Brasil, 2020) e abordado também por Jothimani et al (2020).

Com relação a evolução e manejo dos pacientes hospitalizados, tanto os pacientes com e sem DHC infectados com SARS-COV-2, todos os estudos analisados utilizaram medicações e terapias indicadas internacionalmente e nacionalmente, em consonância com as diretrizes do Centers for Disease Control and Prevention (CDC), Associação Brasileira de Medicina Intensiva Brasileira, Sociedade Brasileira de Infectologia e Sociedade Brasileira de Pneumologia e Tisiologia, as quais não indicam tratamento profilático com quaisquer medicações. Ademais, seguindo as Diretrizes da Sociedade Brasileira de Infectologia, os antibióticos só foram considerados na terapia intensiva em pacientes com suspeita ou confirmação de coinfecção bacteriana, condizentes com os resultados laboratoriais.

Portanto, o uso terapêutico de oxigenoterapia, ventilação mecânica invasiva, antibioticoterapia, corticoterapia, imunoglobulinas humanas e outras medicações elencadas pelos estudos foram assertivas. Entretanto, cabe ressaltar a necessidade da avaliação individual do paciente hospitalizado com DHC infectado com COVID-19, dado que algumas medicações podem ser hepatotóxicas e induzir um quadro de doença hepática medicamentosa (Zhan et al., 2020).

Durante a atual pandemia da COVID-19, há relatos de pacientes adultos hospitalizados com DHC infectados com COVID-19 com apresentação de elevação de transaminases, trombocitopenia, hipoalbuminemia e a alterações na razão de neutrófilos e linfócitos, os quais estão correlacionados com um pior prognóstico do paciente (Gómez et al., 2020; Li et al., 2020). Estas apresentações diagnósticas e laboratoriais estão intimamente relacionadas com a mortalidade dos pacientes evidenciadas neste estudo, tendo variação média entre $1,78 \%$ a $8,1 \%$ para pacientes acima de 18 anos. Outro fator relevante abordado foi o de etnia, dado que Wang et al. (2020) revela que pacientes afro-americanos com DHC infectados com COVID19 apresentaram mortalidade 2x maior que a população caucasiana dos Estados Unidos da América.

Por fim, mesmo com resultados satisfatórios apresentados, este presente estudo possui limitações. Principalmente associadas ao pequeno número na incidência de pacientes adultos hospitalizados com DHC infectados com COVID-19 e poucas pesquisas publicadas nesta população chave. Além do mais, os estudos até o momento publicados apresentam poucos dados quantitativos quanto ao perfil clínico, prevalência e mortalidade dessa população em comparação a outras. Desta forma, o pequeno número de publicações relevantes com dados quantitativos e qualitativos desta população podem interferir na replicação dos resultados deste estudo para trabalhos maiores. No entanto, devido a grande epidemiologia de DHC na população mundial e quadro pandêmico da COVID-19 instaurados, é de suma importância que novas pesquisas com trabalhos epidemiológicos e de estudo de corte para que possam subsidiar ações em políticas públicas, direcionar ações e protocolos de saúde.

\section{Conclusão}

Dada a epidemiologia da DHC na população mundial e quadro pandêmico da COVID-19, pacientes adultos hospitalizados com DHC infectados com COVID-19 podem apresentar quadros de imunossupressão que ocasionam apresentação mais severa da COVID-19 e maior mortalidade quando comparado com grupo de pacientes hospitalizados sem

DHC. Com relação ao perfil clínico, observou-se uma maior frequência do sexo masculino, idade elevada, obesidade, apresentação de doença hepática gordurosa não alcoólica nos pacientes hospitalizados com DHC infectados com SARS-COV2.

No entanto, percebe-se que a população analisada nos artigos é pequena e os dados ainda não são conclusivos, com muitos dados qualitativos e poucos quantitativos, desta forma há a necessidade de novos estudos de corte ou ensaios clínicos 
Research, Society and Development, v. 10, n. 4, e33010413143, 2021 (CC BY 4.0) | ISSN 2525-3409 | DOI: http://dx.doi.org/10.33448/rsd-v10i4.13143

randomizados para elucidar a temática e estabelecer novos manejos e condutas para esta população. No mais, é essencial que os profissionais de saúde estejam atualizados e capacitados para resolução efetiva do problema.

\section{Referências}

Barry, A., Apisarnthanarax, S., O'Kane, G. M., Sapisochin, G., Beecroft, R., Salem, R., ... \& Hawkins, M. A. (2020). Management of primary hepatic malignancies during the COVID-19 pandemic: recommendations for risk mitigation from a multidisciplinary perspective. The Lancet Gastroenterology \& Hepatology.

Gautret, P., Million, M., Jarrot, P. A., Camoin-Jau, L., Colson, P., Fenollar, F., ... \& Raoult, D. (2020). Natural history of COVID-19 and therapeutic options. Expert Review of Clinical Immunology, 1-24.

Gómez, J. L., Aldana, A. J. G., \& Mantilla, M. L. T. (2020). Implicaciones hepáticas en la pandemia por COVID-19. Revista Colombiana de Gastroenterología, 35(Supl. 1), 30-36.

Grasselli, G., Zangrillo, A., Zanella, A., Antonelli, M., Cabrini, L., Castelli, A., ... \& Pesenti, A. (2020). Baseline characteristics and outcomes of 1591 patients infected with SARS-CoV-2 admitted to ICUs of the Lombardy Region, Italy. Jama, 323(16), 1574-1581.

Ji, D., Zhang, D., Yang, T., Mu, J., Zhao, P., Xu, J., ... \& Lau, G. (2020). Effect of COVID-19 on patients with compensated chronic liver diseases. Hepatology international, 14(5), 701-710.

Jothimani, D., Venugopal, R., Abedin, M. F., Kaliamoorthy, I., \& Rela, M. (2020). COVID-19 and Liver. Journal of hepatology.

Koche, J. C. (2011). Fundamentos de metodologia científica: teoria da ciência e iniciação à pesquisa. 1 ed. Petrópolis, RJ: Vozes.

Kovalic, A. J., Satapathy, S. K., \& Thuluvath, P. J. (2020). Prevalence of chronic liver disease in patients with COVID-19 and their clinical outcomes: a systematic review and meta-analysis. Hepatology international, 1-9.

Li, C., Chen, Q., Wang, J., Lin, H., Lin, Y., Lin, J., ... \& Yang, Z. (2020). Clinical characteristics of chronic liver disease with coronavirus disease 2019 (COVID-19): a cohort study in Wuhan, China. Aging (Albany NY), 12(16), 15938.

Mantovani, A., Beatrice, G., \& Dalbeni, A. (2020). Coronavirus disease 2019 and prevalence of chronic liver disease: A meta-analysis. Liver International, 40(6), 1316-1320.

Moon, A. M., Singal, A. G., \& Tapper, E. B. (2019). Contemporary epidemiology of chronic liver disease and cirrhosis. Clinical Gastroenterology and Hepatology.

Pereira, A. S., Shitsuka, D. M., Parreira, F. J., \& Shitsuka, R. (2018). Metodologia da pesquisa científica.[e-book]. Santa Maria. Ed (pp. 3-9). UAB/NTE/UFSM. https://repositorio. ufsm. br/bitstream/handle/1/15824/Lic_Computacao_Metodologia-Pesquisa - Cientifica. pdf.

Richardson, S., Hirsch, J. S., Narasimhan, M., Crawford, J. M., McGinn, T., Davidson, K. W., ... \& Zanos, T. P. (2020). Presenting characteristics, comorbidities, and outcomes among 5700 patients hospitalized with COVID-19 in the New York City area. Jama, 323(20), 2052-2059.

Stasi, C., Fallani, S., Voller, F., \& Silvestri, C. (2020). Treatment for COVID-19: An overview. European Journal of Pharmacology, 173644.

Teich, V. D., Klajner, S., Almeida, F. A. S. D., Dantas, A. C. B., Laselva, C. R., Torritesi, M. G., ... \& Cendoroglo Neto, M. (2020). Características epidemiológicas e clínicas dos pacientes com COVID-19 no Brasil. Einstein (São Paulo), 18.

Wang, Q., Davis, P. B., \& Xu, R. (2020). COVID-19 risk, disparities and outcomes in patients with chronic liver disease in the United States. EClinicalMedicine, 100688 .

Xiao, Y., Pan, H., She, Q., Wang, F., \& Chen, M. (2020). Prevention of SARS-CoV-2 infection in patients with decompensated cirrhosis. The Lancet Gastroenterology \& Hepatology, 5(6), 528-529.

Zhan, K. et al., (2021). Risk factors in patients with COVID-19 developing severe liver injury during hospitalisation. Gut, 70(3), 628-62 